\title{
Laika: A 5uW programmable LSTM accelerator for always-on keyword spotting in 65nm CMOS
}

\author{
J.S.P Giraldo * and Marian Verhelst * \\ *Email: $\{$ sebastian.giraldo, marian.verhelst\}@esat.kuleuven.be \\ *ESAT-MICAS - KU Leuven, Kasteelpark Arenberg 10, B-3001 Leuven, Belgium
}

\begin{abstract}
The ubiquitous importance of speech recognition for diverse applications in mobile devices, necessitates its low power embedded execution. Often, a Keyword Spotting System (KWS) is used to detect specific wake-up words spoken by a user, as a simple user interface, or front-end layer to a larger speech recognition system. Yet, such KWS must be always active, hence imposing strict power and latency constraints. While deep learning algorithms like Long Short-Term Memory (LSTM) demonstrated excellent KWS accuracies, current implementations fail to fit in the tight embedded memory and power budgets.

This paper presents Laika: the implementation of a KWS system using an LSTM accelerator designed in $65 \mathrm{~nm}$ CMOS. For this application, an LSTM model is trained through a speech database and deployed on our custom, yet highly programmable LSTM accelerator. Approximate computing techniques further reduce power consumption, while maintaining high accuracy and reliability. Experimental results demonstrate a power consumption of less than $5 \mu \mathrm{W}$ for real-time KWS applications.
\end{abstract}

Index Terms-LSTM, Keyword Spotting, Deep Learning, Speech Recognition, Digital Accelerator.

\section{INTRODUCTION}

The rise of mobile devices has increased the importance of interactive human-machine interfaces to improve user experience. Voice command recognition, which allows a total handsfree experience for the user, has been subject of continuous research during the last years. For power consumption reasons, a large and more complex speech recognition engine is typically complemented with a more efficient keyword spotting (KWS) wake-up system. Since the KWS system must be always on, its total system power consumption is of utmost importance, required to be well below $100 \mu \mathrm{W}$ for continuous operation.

Along with this trend, Deep Learning has shown impressive results in terms of accuracy for different speech recognition tasks including keyword spotting [1]. These typically make use of recurrent neural networks, such as LSTMs, which allow to capture dynamic temporal behavior, yet pose a significant computational burden on the embedded device. To lower this burden, hardware accelerators have been developed targeting Keyword Spotting. [2] shows an ASIC with 8 execution engines for accelerating RNNs, showing its application for realtime KWS, consuming $6.6 \mathrm{~mW}$ at $20 \mathrm{MHz} / 0.8 \mathrm{~V}$. A compact memory storage is presented in [3] through a programmable processor with $270 \mathrm{kB}$ on-chip weight which executes realtime KWS with $300 \mu \mathrm{W}$ power consumption. In [4] a SIMD processor evaluates DNNs for automatic speech recognition tasks including a small vocabulary recognizer which achieves
$172 \mu \mathrm{W}$ for real time power consumption. All these accelerators target the support of medium-to-large size deep learning models. They however fail to achieve real time operation with power budgets in the order of $10 \mu \mathrm{W}$. For example, in [3] just leakage power accounts for more than $20 \mu \mathrm{W}$ of the total power consumption.

Yet, recent work [5] [1], indicates that a novel type of networks, Long Short-Term Memory networks (LSTMs), can attain acceptable KWS accuracies with only small sized networks. The customized acceleration of small but cheap LSTM models has remained unexplored, although its potential for reliable keyword spotting systems is eminent. Its realization is challenged by the careful balance needed between task accuracy and power consumption, necessitating cross-layer optimizations between the algorithm and hardware implementations, without giving up flexibility and programmability.

In this work we show such co-optimization together with the implementation of Laika (LSTM Accelerator IC for KWS Analysis), a low power, programmable LSTM accelerator in $65 \mathrm{~nm}$ optimized for Keyword Spotting. The chip can execute small-medium size LSTMs, with support for different network topologies, enabling to broaden the application to other domains. Power consumption is kept low through several cross-layer $\mathrm{HW}$-algorithm co-optimizations, and by keeping the full model storage on-chip, avoiding any high speed I/O. Our contribution can be summarized as follows:

- Extensive HW-algorithm co-optimization for always-on LSTM operation jointly exploiting linear and non-linear quantization and activation function approximation.

- A flexible, programmable implementation with support for diverse LSTM networks and topologies, capable of storing complete models on chip.

- The first LSTM accelerator which can support always-on real-time keyword spotting, consuming less than $10 \mu \mathrm{W}$.

In Section II the theoretical background for LSTMs is presented. Section III details the software/hardware co-design to reduce model size and computational complexity. In Section IV, the system architecture is discussed, highlighting the data flow and Laika's micro-architecture. The chip's measurement results are highlighted in Section V, and Section VI summarizes this work and compares it to the state-of-the-art. 


\section{LSTMS FOR KEYWORD SPOTTING}

\section{A. LSTMs}

LSTMs, which stands for Long Short-Term Memory, are a more complex type of recurrent neural network that show state-of-the-art accuracy for sequence tasks like speech recognition and natural language processing. Every layer of an LSTM consists of a set of neurons, each computing 4 intermediate results called 'gates' (forget $f_{t}$, input $i_{t}$, output $o_{t}$ and candidate gate $g_{t}$ ), from an internally stored hidden vector $h_{t-1}$ and the current input vector $x_{t}$ :

$$
\begin{gathered}
f_{t}=\sigma_{s}\left(W_{f} x_{t}+U_{f} h_{t-1}+b_{f}\right) \\
i_{t}=\sigma_{s}\left(W_{i} x_{t}+U_{i} h_{t-1}+b_{i}\right) \\
o_{t}=\sigma_{s}\left(W_{o} x_{t}+U_{o} h_{t-1}+b_{o}\right) \\
g_{t}=\sigma_{h}\left(W_{g} x_{t}+U_{g} h_{t-1}+b_{g}\right) \\
c_{t}=f_{t} \circ c_{t-1}+i_{t} \circ g_{t} \\
h_{t}=o_{t} \circ \sigma_{h}\left(c_{t}\right)
\end{gathered}
$$

where $W_{*}, U_{*}$ and $b_{*}$ are trained model coefficients for each gate, and $\sigma_{*}$ are nonlinear activation functions tanh $\sigma_{h}$ and sigmoid $\sigma_{s}$. The result of these gates (1)-(4) is subsequently used to compute the next memory cell $c_{t}$ and hidden vector $h_{t}$ through a series of element-wise multiplications, denoted by $\circ$ in (5)-(6).

LSTM models typically consist out of one to multiple of such LSTM layers, followed by one or more fully connected layers, operating on the outputs $h_{t}$ of the last LSTM layer:

$$
z_{t}=W_{f c} h_{t}
$$

where $W_{f c}$ are weights for the fully connected layer. As LSTMs are capable of achieving good accuracies on problems with dynamic temporal behavior with relatively smaller model sizes compared with other feed-forward neural networks such as CNNs, it makes them excellent candidates for KWS systems.

\section{B. Keyword Spotting}

Keyword Spotting typically relies on a pipeline of a feature extraction module, followed by a keyword classifier. Commonly used features for speech recognition applications are Mel Frequency Cepstral Coefficients (MFCC). These features are extracted from windows of audio samples, typically 2532 ms windows, with a $10-16$ ms overlap between subsequent windows. For every window, the extracted set of MFCC coefficients is subsequently sent to the classifier to detect target keywords.

In this work, we rely on such MFCC features, and further optimize the classification stage. Recent work has shown that it is possible to implement accurate, yet compact KWS using small-scale LSTMs [5] showing good results with a single LSTM layer with up to 64 neurons [1]. It is however important to realize that research to further balance accuracy and complexity is still in progress. As such, any hardware implementation hence has to maintain good programmability regarding the number of LSTM and fully connected layers.
TABLE I

IMPACT APPROXIMATE COMPUTING ON KWS ACCURACY

\begin{tabular}{|c|c|c|c|}
\hline Numerical Precision & Precision & Recall & F1-score \\
\hline FP & 0.920 & 0.890 & 0.904 \\
\hline FxP without PLA & 0.904 & 0.888 & 0.896 \\
\hline FP with PLA & 0.920 & 0.889 & 0.904 \\
\hline FxP with PLA & 0.918 & 0.882 & 0.900 \\
\hline NLQ without fine-tuning & 0.910 & 0.805 & 0.853 \\
\hline NLQ with fine-tuning & 0.917 & 0.883 & 0.900 \\
\hline
\end{tabular}

\section{Algorithm-HaRdware Co-OPtimization}

A hardware accelerator for real-time execution of such LSTMs should be able to consume a new feature vector every $16 \mathrm{~ms}$, at low power consumption, without giving up programmability. Several algorithm-hardware co-optimizations have been performed with the goal of 1.) reducing the memory footprint of the network model, enabling efficient on-chip storage of the full model; 2.) reducing the computational complexity of the required model execution, enabling low power datapath operation.

All optimizations were assessed on a keyword spotting system consisting of a 56 neuron LSTM layer followed by one fully connected (FC) layer similar to [5]. The network was first trained in floating point, using the TIMIT database and a segmentation of $500 \mathrm{~ms}$ for labeling. The network is fed with 39 MFCC features per $32 \mathrm{~ms}$ window with $16 \mathrm{~ms}$ overlap. The four most common words of the dataset (water, greasy, wash and dark) were chosen as KWS targets and a binary classifier was trained for each of them, resulting in the floating point (FP) baseline accuracy for the test set shown in Table I. The Table presents the average precision, recall and F1 score (harmonic mean between precision and recall).

a) Network quantization: The use of low precision operations and model coefficients significantly reduces computational complexity and memory bandwidth with marginal loss in accuracy. The obtained 32-bits floating point parameters and inputs can be quantized to a low-resolution fixed point representation. Table I shows that accuracy is nearly unaltered when scaling down to 8-bit fixed point operation (FxP without Piecewise Linear Approximation) for inputs and weights, with internal 16-bit multiplication results, summed in 32-bit accumulators before being stored back into 8-bit registers capturing the outputs of the LSTM layer.

b) Function approximation: LSTMs require several nonlinear activation functions tanh $\sigma_{h}$ and sigmoid $\sigma_{s}$, which are expensive to compute exactly. They can however be approximated through a piecewise linear function relying on hardware LUTs storing the coefficients $m$ and $b$ of each segment of the linear approximations $m x+b$. Yet, the number of segments has to be selected carefully to balance hardware cost vs. accuracy implications. As can be seen in the aforementioned table (FP, resp. FxP with PLA), 8 linear segments still allow to obtain good accuracy, while enabling very small LUTs.

c) Non-linear quantization: To further reduce precision below 8-bit, without giving up accuracy, clustering techniques similar to Deep Compression [6] can be applied. By cluster- 
ing 8-bit model weights into $2^{M}$ representative values, only $M$-bits instead of 8-bits are needed for coefficient storage. Online, each $M$-bit word is translated back to its 8-bit cluster center value using a small codebook look up table (LUT), before using it in the LSTM computation. This non-linear quantization of the model weights into $2^{M}$ clusters however reduces detection performance, as can be seen in Table I (NLQ without fine-tuning, $M=4)$. Yet, the original accuracy can be recovered by retraining the cluster centers after clustering, showing good results for $\mathrm{M}=4$ (NLQ with fine-tuning). The LSTM coefficients can now be stored in on-chip memory using only 4-bits each, and be converted back to their 8-bit cluster center value in a LUT when fetched for computation. All hardware-algorithm co-optimizations together as such reduce the memory footprint with up to $8 \mathrm{x}$ and simplify the computation of network gates and activation functions, while showing negligible loss compared to a 32-bits FP model.

\section{HARDWARE ARCHITECTURE}

To exploit the discussed algorithmic optimizations, a custom yet highly-programmable hardware accelerator was developed for Laika.

a) System architecture: The system architecture is depicted in figure 1 . The near-memory compute processor avoids any off-chip data communication besides the MFCC inputs and KWS detection outputs. A $32 \mathrm{kB}$ on-chip memory stores the complete LSTM+FC model, while internal data buffers buffer data close to the Functional Units (FUs). Four processing elements (PE) each are responsible for one gate of an LSTM neuron (eqn. (1)-(4)). They are fed the required inputs from 3 possible storage locations: 1.) the 8-bits model coefficients $W_{*}$ and $U_{*}$ from the Model Memory; 2.) the MFCC input data $x_{t}$ from the input buffer; and 3.) the hidden state $h_{t-1}$ from the data buffers, with support for up to 2 LSTM layers (corresponding to the FIFOs $H 1$ and $H 2$ in the architecture).

Every PE performs the required 2 multiplications per gate, which is accumulated in a 32-bits value (see fig. 2). After cycling through the multiply-and-accumulate operations of each neuron (corresponding to a complete row of the gate matrices in eqn. (1)-(4)) the accumulation output is fed to the activation function (NL), implemented with the 8-segment LUT-based function approximation. Two LUT blocks per function (one saving the slope $m$ and the other the offset $b$ for each linear approximation $m x+b$ ) are shared by all 4 PE's. The output of the non-linear functions (NL OUT 1 4 ) is subsequently sent to the gate buffers, formed by 8-bit FIFOs implemented with registers: one for each of the gates $(I, G, F, O)$.

After computing the gates, all the element-wise multiplications are carried out by one PE. This PE loads data from the gate buffers, the hidden state buffer $(H 1$ or $H 2)$ and memory cell buffer $(C 0$ or $C 1)$ to compute a new hidden state that is saved into the correspondent hidden state buffer (eqn(5)-(6)). In order to execute the FC layers, one PE loads the weights from the Model Memory and data from the hidden state buffers ( $H 1$ or $H 2$ ), computing the multiply-and-accumulate operations for each output neuron and saving the result into the output buffer as final products of the frame inference (eqn. (7)).

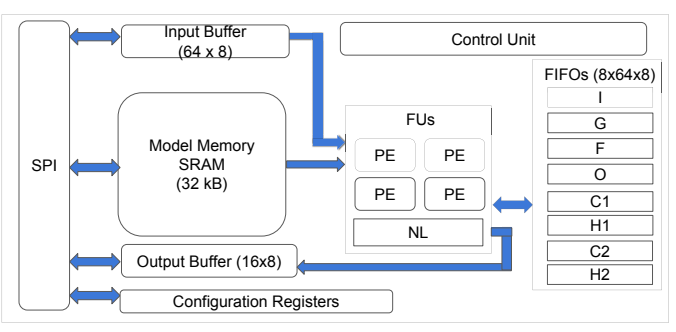

Fig. 1. Top level chip architecture

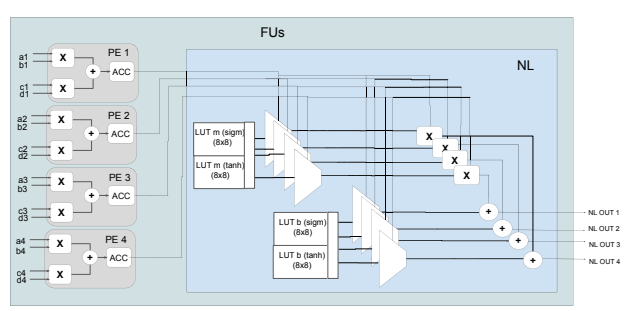

Fig. 2. Microarchitecture of the PEs and non-linear approximator NL.

b) Clustering mode: Laika supports nonlinear quantization, through programmable LUTs which can be activated between the model memory and the PEs. In this mode, 4-bit model weights are fetched from memory, doubling the amount of fetched weights per clock cycle. While the first half of the weights is subsequently sent to the LUT, decoding the 4bit cluster values into the actual 8-bit weights, the other half is buffered to be used the next clock cycle. This effectively halves the memory bandwidth, positively impacting system level power consumption as will be shown in Section V. A different LUT is used for each gate, allowing to train cluster values for each gate independently.

c) Accelerator programmability: A control unit controls the operation of the PEs and memory fetches. It can be configured to execute various network topologies through a series of configuration registers (number of hidden LSTM layers, number of neurons per layers, clustering en/disabled), as well as the dimensions of the FC layer (dictating the number of targeted detection keywords). An SPI interfaces with these configuration data, the model memory, the input buffer, output buffer and the internal FIFO buffers. Reprogramming model memory and configuration registers allows support for a wide variety of applications and power-accuracy trade-off points.

\section{IMPLEMENTATION AND MEASUREMENTS}

a) Physical implementation: The chip, depicted in Fig. 3 , is implemented in $65 \mathrm{~nm}$ CMOS with a standard cell P\&R flow. To further improve power efficiency while ensuring reliability, several back-end optimizations were implemented: Logic and SRAM power domains were separated to allow maximal voltage scaling for each domain. Aggressive clock 
gating was applied through a global 'freeze' input signal, which gates the clock tree of the complete data path to stall the system when the accelerator completes an inference before the next real time feature vector arrives. Finally, high Vt standard cells were used for logic ensuring low leakage, with low $\mathrm{Vt}$ standard cells for clock distribution to allow more aggressive voltage scaling without generating hold violations.

b) Measurement setup: A ZedBoard Zynq-7000 ARM/FPGA interfaces over SPI with the configuration registers, model weights, input features and detection outputs. Since Laika supports the storage of a complete model onchip, only feature vectors and detection outputs are actually communicated during real-time measurements.

c) Performance measurements: Fig 3 shows the measured minimum supply voltage for the accelerator logic at various operating frequencies, fixing SRAM voltage to $0.7 \mathrm{~V}$. The system is able to work with voltages in the range of $0.575 \mathrm{~V}-0.8 \mathrm{~V}$ at clock frequencies ranging $380 \mathrm{kHz}-8 \mathrm{MHz}$. The figure moreover shows the resulting measured energy consumption for a complete KWS inference on a $16 \mathrm{msec}$ frame. The energy of the complete system (including memories) is measured when deploying the 56-neuron KWS model of Section III, for both linear quantization (LQ) and nonlinear quantization (NLQ). The energy per inference improves with lower frequencies because of voltage scaling, with a floor due to leakage power. Nonlinear quantization reduces the energy per inference with $25 \%$, due to reduced memory fetching.
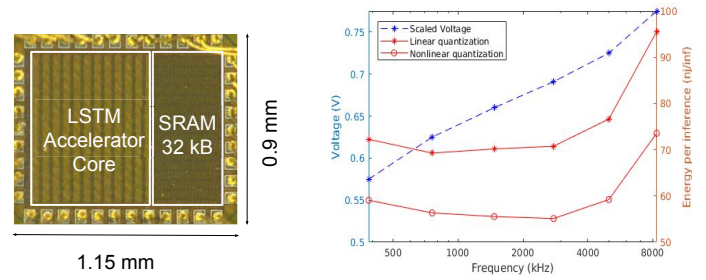

Fig. 3. Left) Photo of chip. Right) Maximal Operating Frequency for different voltages and Energy Efficiency for several operating points.

d) Application and flexibility measurements: Power consumption was measured when deploying different network topologies in a real-time keyword spotting application. The features are fed to the accelerator at a constant rate of $16 \mathrm{~ms}$. The clock frequency and supply voltage of the chip are adjusted to match the required computation speed for such real time operation. This information is summarized in Table II (with $h l$ the number of hidden layers and $n$ the number of neurons per layer, all followed by 1 FC layer). All deployed networks fit in the $32 \mathrm{kB}$ SRAM. The power consumption lies between $2-7 \mu \mathrm{W}$ depending on the model topology. Using the model optimized in Section III, real-time KWS can be achieved with $<5 \mu \mathrm{W}$ at $0.575 \mathrm{~V} / 250 \mathrm{kHz}$ by activating the nonlinear quantization mode.

\section{CONCLUSIONS}

This paper presents Laika, an ultra-low power LSTM accelerator for Keyword Spotting. The chip supports full on-
TABLE II

REAL-TIME POWER CONSUMPTION FOR DifFERENT NeTWORK TOPOLOGIES

\begin{tabular}{|c|c|c|c|c|c|}
\hline Model & Freq(kHz) & MACs & $\begin{array}{c}\text { Leakage } \\
(\mu \mathbf{W})\end{array}$ & $\begin{array}{c}\text { Total Power } \\
\text { LQ }(\mu \mathbf{W})\end{array}$ & $\begin{array}{c}\text { Total Power } \\
\text { NLQ }(\mu \mathbf{W})\end{array}$ \\
\hline $1 \mathrm{hl}, 40 \mathrm{n}$ & 148 & 12800 & 0.614 & 3.84 & 3.23 \\
\hline $1 \mathrm{hl}, 48 \mathrm{n}$ & 196 & 16896 & 0.614 & 4.80 & 4.08 \\
\hline $1 \mathrm{hl} 56 \mathrm{n}$ & 250 & 21504 & 0.614 & 5.85 & 4.97 \\
\hline $1 \mathrm{hl}, 64 \mathrm{n}$ & 309 & 26624 & 0.614 & 7.22 & 6.01 \\
\hline $2 \mathrm{hl}, 16 \mathrm{n}$ & 77 & 5888 & 0.614 & 2.27 & 2.05 \\
\hline $2 \mathrm{hl}, 24 \mathrm{n}$ & 147 & 11328 & 0.614 & 3.70 & 3.27 \\
\hline $2 \mathrm{hl}, 32 \mathrm{n}$ & 239 & 18432 & 0.614 & 5.65 & 4.91 \\
\hline $2 \mathrm{hl}, 40 \mathrm{n}$ & 352 & 27200 & 0.614 & 7.98 & 6.84 \\
\hline
\end{tabular}

TABLE III

PERFORMANCE SUMMARY AND COMPARISON WITH STATE-OF-THE-ART

\begin{tabular}{|c|c|c|c|c|}
\hline & $\begin{array}{c}\text { This } \\
\text { work }\end{array}$ & $\begin{array}{c}2017 \text { ISSCC } \\
\text { Price [4] }\end{array}$ & $\begin{array}{c}2017 \text { ISSCC } \\
\text { Bang [3] }\end{array}$ & $\begin{array}{c}\text { 2017 ESSCIRC } \\
\text { Chen [2] }\end{array}$ \\
\hline Technology & $65 \mathrm{~nm}$ & $65 \mathrm{~nm}$ & $40 \mathrm{~nm}$ & $65 \mathrm{~nm}$ \\
\hline ML algorithm & LSTM & DNN & DNN & GRU \\
\hline Area [mm2] & $1.15 \times 0.9$ & $3.1 \times 3.1$ & $2.5 \times 2.85$ & $2.9 \times 3.5$ \\
\hline $\begin{array}{c}\text { On-chip } \\
\text { memory }\end{array}$ & $32 \mathrm{kB}$ & $5.84 \mathrm{Mb}$ & $270 \mathrm{kB}$ & $64 \mathrm{kB}$ \\
\hline $\begin{array}{c}\text { Power for } \\
\text { real-time KWS }\end{array}$ & $5 \mu \mathrm{W}$ & $172 \mu \mathrm{W}$ & $321 \mu \mathrm{W}$ & $6.6 \mathrm{~mW}$ \\
\hline Clock & $250 \mathrm{kHz}$ & $3 \mathrm{Mhz}$ & $3.9 \mathrm{MHz}$ & $20 \mathrm{Mhz}$ \\
\hline Voltage & 0.575 & 0.6 & 0.65 & 0.8 \\
\hline Energy per inf. & $60 \mathrm{~nJ}$ & $4.5 \mu \mathrm{J}$ & $2 \mu \mathrm{J}$ & NA \\
\hline
\end{tabular}

chip models through a $32 \mathrm{kB}$ SRAM, allowing to execute small-medium size LSTM networks with a broad variety of programmable topologies. The accelerator is able to execute models using linear as well as nonlinear quantization. The support for clustered models allows to improve the energy efficiency of inference without impacting accuracy. The prototype achieves between $59-94 \mathrm{~nJ} /$ inference at $380 \mathrm{kHz}-8 \mathrm{Mhz}$. For a real-time Keyword Spotting application the accelerator consumes less than $5 \mu \mathrm{W}$ at $0.575 \mathrm{~V}$.

\section{REFERENCES}

[1] M. Sun, A. Raju, G. Tucker, S. Panchapagesan, G. Fu, A. Mandal, S. Matsoukas, N. Strom, and S. Vitaladevuni, "Max-pooling loss training of long short-term memory networks for small-footprint keyword spotting," in 2016 IEEE Spoken Language Technology Workshop (SLT), Dec 2016, pp. $474-480$.

[2] C. Chen, H. Ding, H. Peng, H. Zhu, R. Ma, P. Zhang, X. Yan, Y. Wang, M. Wang, H. Min, and R. C. J. Shi, "Ocean: An on-chip incrementallearning enhanced processor with gated recurrent neural network accelerators," in ESSCIRC 2017 - 43rd IEEE European Solid State Circuits Conference, Sept 2017, pp. 259-262.

[3] S. Bang, J. Wang, Z. Li, C. Gao, Y. Kim, Q. Dong, Y. P. Chen, L. Fick, X. Sun, R. Dreslinski, T. Mudge, H. S. Kim, D. Blaauw, and D. Sylvester, "14.7 a 288 uw programmable deep-learning processor with $270 \mathrm{~kb}$ onchip weight storage using non-uniform memory hierarchy for mobile intelligence," in 2017 IEEE International Solid-State Circuits Conference (ISSCC), Feb 2017, pp. 250-251.

[4] M. Price, J. Glass, and A. P. Chandrakasan, "14.4 a scalable speech recognizer with deep-neural-network acoustic models and voice-activated power gating," in 2017 IEEE International Solid-State Circuits Conference (ISSCC), Feb 2017, pp. 244-245.

[5] P. Baljekar, J. F. Lehman, and R. Singh, "Online word-spotting in continuous speech with recurrent neural networks," in 2014 IEEE Spoken Language Technology Workshop (SLT), Dec 2014, pp. 536-541.

[6] S. Han, X. Liu, H. Mao, J. Pu, A. Pedram, M. A. Horowitz, and W. J. Dally, "Eie: Efficient inference engine on compressed deep neural network," in 2016 ACM/IEEE 43rd Annual International Symposium on Computer Architecture (ISCA), June 2016, pp. 243-254. 